\title{
Impact of pelvic magnetic resonance imaging findings in the indication of uterine artery embolization in the treatment of myoma
}

\author{
Norma Maria Tenório Brito Pires ${ }^{1}$, Emmanuelle Tenório Godoi ${ }^{1}$, Dinaldo Cavalcanti Oliveira ${ }^{1}$, \\ Simone Cristina Soares Brandão ${ }^{1}$, Carlos Coutinho Abath ${ }^{1}$, Pedro Pires ${ }^{2}$, Edward Araujo Júnior ${ }^{3}$ \\ ${ }^{1}$ Science Health Center, Federal University of Pernambuco, Recife, Brazil \\ ${ }^{2}$ Department of Maternal and Child, University of Pernambuco, Recife, Brazil \\ ${ }^{3}$ Department of Obstetrics, Paulista School of Medicine — Federal University of São Paulo, Brazil
}

\begin{abstract}
Objectives: To assess the impact of pelvic magnetic resonance imaging (MRI) findings in the indication for uterine-artery embolization in women with fibroids, as well as the correlation between MRI and ultrasound (US) examinations for diagnosing adenomyosis.

Material and methods: A retrospective observational study was performed through the analysis of the medical records of 263 women referred for uterine-artery embolization as treatment for fibroids after undergoing US and MRI examinations. To compare uterine volume and fibroid measurement in US and MRI, the Wilcoxon test was used; for the number of fibroids, the McNemar test was used. The kappa coefficient was used to evaluate the correlation between US and MRI findings for diagnosing adenomyosis.

Results: The mean age of patients was $37.9 \pm 6.8$ years and 191 (72.6\%) were nulliparous. Forty-three patients with adenomyosis associated with fibroid were diagnosed by MRI; US indicated the presence of adenomyosis in $12(4.56 \%)$ women. There was agreement between MRI and US in the diagnosis of adenomyosis in 218/263 (82.9\%) patients $(p<0.05)$. In the US examination, the mean uterine volume was lower $\left(389 \pm 340.8 \mathrm{~cm}^{3}\right)$ than that observed in MRI $\left(472.2 \pm 415.9 \mathrm{~cm}^{3} ; \mathrm{p}<0.001\right)$. Regarding the number of fibroids, MRI showed a greater number of patients with multiple fibroids $(68.8 \% \mathrm{vs.} 57.4 \%$, MRI and US, respectively; $\mathrm{p}<0.001$ ).
\end{abstract}

Conclusions: In women with fibroids referred for uterine-artery embolization, MRI findings led to the revision of the initial diagnosis in $17.1 \%$ cases. US showed a lower sensitivity for diagnosing adenomyosis than MRI.

Key words: uterine-artery embolization, magnetic resonance imaging, ultrasound, adenomyosis

Ginekologia Polska 2017; 88, 3: 129-133

\section{INTRODUCTION}

Uterine fibroids (myoma) are the most common tumor in the female genital tract, with an estimated incidence of 20-40\% among women of reproductive age, and higher incidence in Black women compared with Caucasians [1]. Although fibroids are often asymptomatic, they can cause various symptoms such as abnormal uterine bleeding, a feeling of heaviness in the lower abdomen, urinary incontinence or retention, and lower abdominal pain $[2,3]$. Hysterec- tomy is considered the definitive treatment and may be performed vaginally, abdominally, or laparoscopically [4]. The need to preserve the uterus and appendages led to the development of less-invasive techniques such as myomectomy [5], which was the standard treatment until the uterine-artery embolization technique was developed [3]. The basic principle of embolization is selective catheterization of the uterine arteries and occlusion of the distal arteries which feed the fibroids, causing tumor infarction

Corresponding author:

Edward Araujo Júnior

Rua Belchior de Azevedo, 156 apto. 111 Torre Vitoria

São Paulo-SP, Brazil

CEP 05089-030

tel./fax: +55 1137965944

e-mail: araujojred@terra.com.br 
with preservation of the uterus $[2,6]$. The other options for the uterine myoma treatment are the following: magnetic resonance-guided high-intensity focused ultrasound (MRgFUS), transvaginal route for radiofrequency ablation, and robotic laparoscopic myomectomy. MRgFUS is comparable to uterine-artery embolization, and appears to be a cost effective treatment option, especially in older women. Transvaginal route for radiofrequency ablation is a promising new nonsurgical alternative, which needs to be studied in larger trials to establish its safety and efficacy. Robotic laparoscopic myomectomy is comparable to traditional laparoscopic technique in short surgical outcomes but is associated with higher costs [7].

Ultrasound (US) is considered the examination of choice for the initial diagnosis and monitoring of uterine fibroids [8]. But because of its multiplanar capacity, excellent tissue contrast, and reproducibility, magnetic resonance imaging (MRI) has become the investigation of choice in cases requiring further investigation [3]. One study showed that when the indication for imaging investigations is to define the best therapeutic strategy, especially in cases selected for uterine-artery embolization, information provided from US imaging is insufficient and may lead to therapeutic errors in up to $25 \%$ cases [9].

\section{OBJECTIVES}

The aim of this study was to assess the impact of pelvic MRI findings in the indication for uterine-artery embolization in women with fibroids, as well as the agreement between MRI and US examinations for diagnosing adenomyosis.

\section{MATERIAL AND METHODS}

This retrospective observational study analyzed the medical records of women referred for uterine-artery embolization to treat fibroids between January 2000 and April 2015, who had undergone US and MRI examinations and for whom medical history was available. The US examinations were performed at several imaging clinics in the city of Recife, Pernambuco, Brazil, and MRI were performed at a single reference center in interventional radiology. Inclusion criteria were aged $>18$ years, and interval of up to 12 months between US and MRI examinations. The exclusion criterion was incomplete medical records. This study was approved by the Ethics Committee of the Federal University of Pernambuco (UFPE); the need for an informed consent was waived because this study was retrospective in nature.

The US examinations were performed using an intracavitary transducer and supplemented by a transabdominal US in cases of bulky uterus. All reports contained information on the appendages and endometrium. For the fibroids, the number, location relative to the myometrium and/or endometrium, and measurements of the largest fibroid were obtained. Uterine volume was measured in $\mathrm{cm}^{3}$, and was calculated using the following formula: longitudinal $\times$ transverse $\times$ anteroposterior $\times 0.52$. US criteria of adenomyosis were the following: (1) uterus enlarged, (2) heterogeneous myometrium with hipoecoic areas, (3) thickening or abnormality of the subendometrial myometrium, (4) linear grooves from endometrium to myometrium, (5) subendometrial retention cysts with variable format or dimension, and (6) asymmetry of uterine wall with predominance of the posterior wall.

The MRI examinations were performed by a single radiologist using a Magnetom Aera 1.5 Atim device (Siemens AG, Erlangen, Germany). The following sequences were initially obtained:T1 showing the edge and the internal anatomy of the organs, and T1 with fat saturation. Next, a MRI contrast agent (gadolinium — diethylene triamine pentaacetic acid) was injected. A new T1 sequence with fat saturation was then performed to distinguish the layers of the uterus and the cancer stages, if present, as well as a T2 sequence to differentiate the uterine layers and permit diagnosis of any abnormalities (Fig. 1). Sagittal, axial and coronal sections of the uterus were obtained, regarding the endometrial cavity. To diagnose adenomyosis, the junctional zone borders were evaluated for uniformity and thickness. Thickness was measured in the anterior and posterior walls at the thickest portion. A diagnosis of adenomyosis was made when the junctional zone was not uniform, in the presence of poorly delimited focal areas in the myometrium, and when the thickness of the junctional zone was $\geq 12 \mathrm{~mm}[10,11]$ (Fig. 2). The following data from the clinical history were analyzed: age, parity, desire to have children in the future, previous treatment, and current symptoms.

The indications for uterine-artery embolization were as follows: (1) vascularized fibroid, (2) unsuccessful surgical approach to treat the fibroid, (3) fibroid associated with adenomyosis, and (4) intramural fibroid of $>5 \mathrm{~cm}$. The contraindications were as follows: (1) non-vascular degenerated fibroids, (2) subserosal or submucosal fibroid with pedicle, (3) absence of fibroid, and (4) presence of adenomyosis not associated with fibroids.

The data were transferred into an Excel 2007 spreadsheet (Microsoft Corp., Redmond, WA, USA) and analyzed using the Statistical Package for the Social Sciences software (SPSS) version 20.0 for Windows (SPSS Inc., Chicago, IL, USA). For the quantitative variables (age, uterine volume, number of fibroids, size of fibroids), data were expressed as means, medians, standard deviations, minimum, and maximum. For qualitative variables (parity, desire to have children in the future), data were expressed as percentages (\%). For the comparison between uterine volume and the extent of fibroid between US and MRI findings, the Wilcoxon test was used; for the number of fibroids, the McNemar test was applied. To 


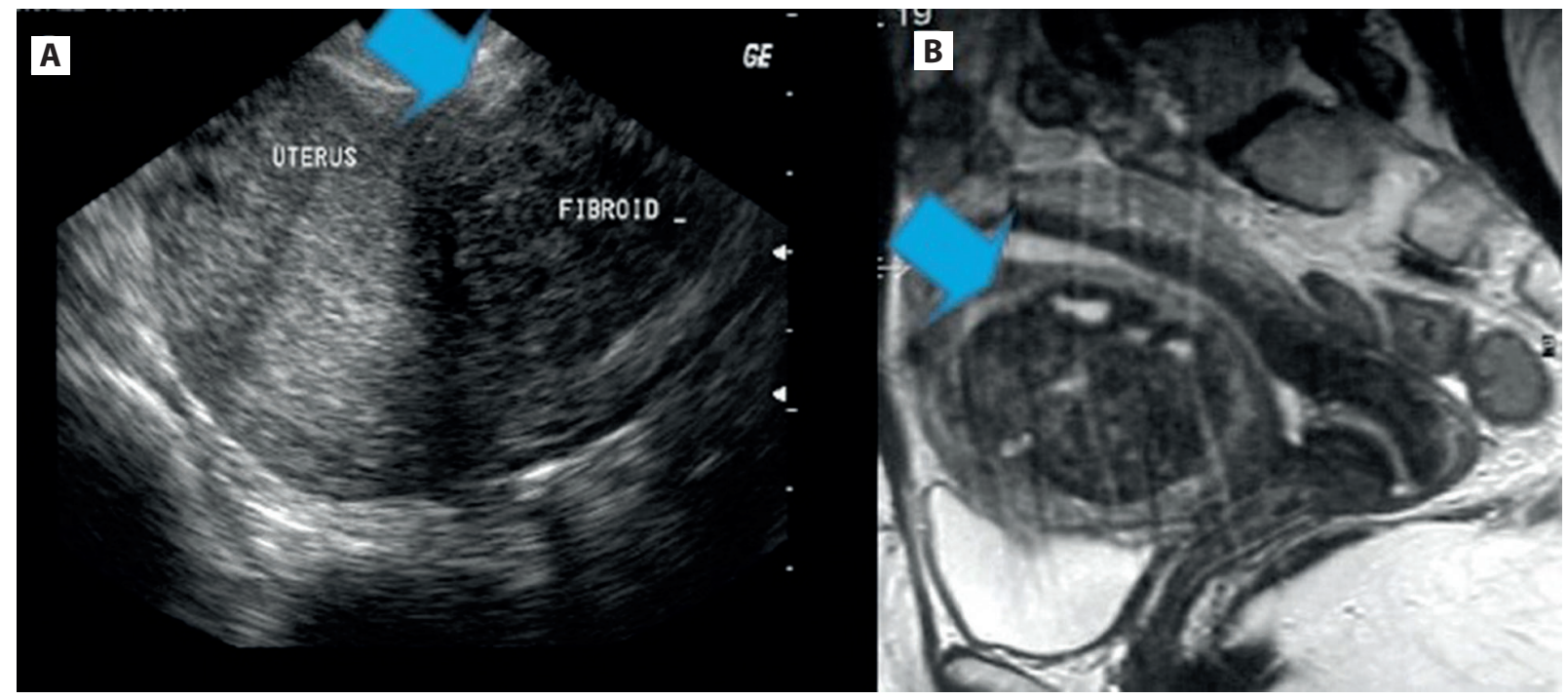

Figure 1.A. Transvaginal ultrasound, sagittal plane, showing uterine fibroid (blue arrow); B. Magnetic resonance imaging, sagittal plane, T1 showing uterine fibroid (blue arrow)

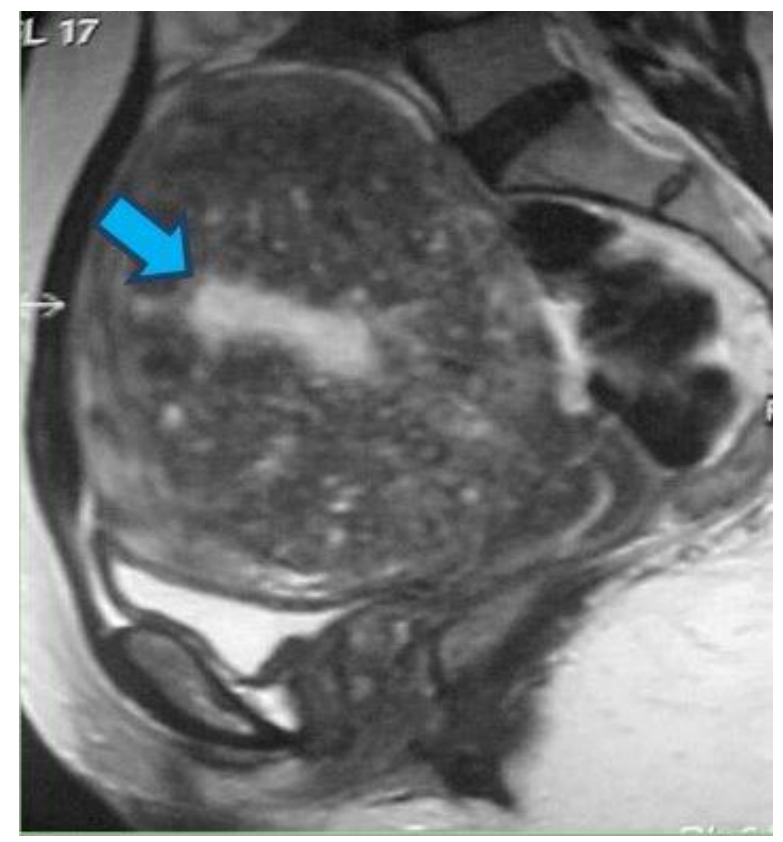

Figure 2. MRI of the pelvis in the sagittal plane, $\mathrm{T} 1$ showing a uterus with adenomyosis with loss of definition of the junctional zone (blue arrow)

assess the correlation between US and MRI findings in the diagnosis of adenomyosis, the kappa coefficient was used, and classified as follows: poor, 0.00-0.20; fair, 0.20-0.40, moderate, 0.40-0.60; good, 0.60-0.80; and very good, 0.80-1.00. In all analyses, a significance level of ( $p$ ) of $<0.05$ was adopted.

\section{RESULTS}

The study included 263 patients with fibroids, mean age $37.9 \pm 6.8$ years (range 18-58 years). As for parity, 191 (72.6\%) were nulliparous; 41 (15.6\%) primiparous; and 31 (25.1\%) multiparous. Of this sample group, 149 (56.7\%) reported the desire to have children. One hundred and twenty two patients referred previous treatment for uterine myoma: hormonal 94/122 (77.0\%), hysteroscopy $27 / 122$ (22.1\%), laparoscopy 14/122 (11.5\%), and laparotomy 28/122 (23.0\%). None of these patients had indication to hysterectomy treatment.

A total of 43 patients with adenomyosis associated with fibroids were diagnosed by MRI, with a mean age of $37.9 \pm 6.8$ years (range 22-58 years); of these, $84 \%$ were aged $>35$ years. As for parity, 25/43 (58\%) were nulliparous; $8 / 43$ (18.6\%) primiparous, and 10/43 (23\%) multiparous. The desire to have children in the future was reported by $15 / 43$ (34.9\%) of these women, 13/15 (86.6\%) of whom were nulliparous. The presence of symptoms was observed in 42/43 (97.7\%) women: uterine bleeding in 31/42 (73.8\%), lower abdominal pain in 6/42 (14.3\%), sensation of increased abdominal volume in 2/42 (4.7\%), increased urinary frequency in $1 / 42(2.4 \%)$, and difficulty conceiving in 2/42 (4.7\%).

In US imaging, mean uterine volume was lower $\left(389 \pm 340.8 \mathrm{~cm}^{3}\right)$ than the volume observed in MRI $\left(472.2 \pm 415.9 \mathrm{~cm}^{3} ; \mathrm{p}<0.001\right)$. The mean size of the largest fibroid was statistically similar between the two methods. Regarding the number of fibroids, MRI showed a greater number of women with multiple fibroids (68.8\% vs. $57.4 \%$, MRI and US, respectively, $\mathrm{p}<0.001$ ) (Tab. 1).

Of the 263 evaluated patients, 216 (82.1\%) had an indication for uterine-artery embolization according to their clinical data and US. After MRI, the indication was altered for 51 (19.3\%) patients. Of the 216 patients initially indicated for embolization, 33 (15.3\%) were contraindicated by MRI results; of the 47 that were contraindicated by US, MRI showed indication in 18 (38.3\%). According to Table 2, 
Table 1. Sonographic and magnetic resonance imaging data of women with adenomyosis referred for uterine-artery embolization

\begin{tabular}{|c|c|c|c|}
\hline Characteristic & Ultrasound & Magnetic resonance imaging & $p$ \\
\hline \multicolumn{4}{|l|}{ Uterine volume $\left[\mathrm{cm}^{3}\right]$} \\
\hline Mean (SD) & $289.2(211.9)$ & $334.9(220.4)$ & \multirow{3}{*}{$0.049^{1}$} \\
\hline Median $\left(25^{\text {th }}-75^{\text {th }}\right)$ & $236.8(118.4-412.9)$ & $297.0(173.0-445.3)$ & \\
\hline Minimum-maximum & $58.6-916$ & $76.0-1.029$ & \\
\hline \multicolumn{4}{|l|}{ Number of fibroids } \\
\hline 1 or 2 fibroids & $19(44.2 \%)$ & $11(25.6 \%)$ & \multirow{2}{*}{$0.021^{2}$} \\
\hline ultiple & $24(55.8 \%)$ & $32(74.4 \%)$ & \\
\hline \multicolumn{4}{|l|}{ Largest fibroid [cm] } \\
\hline Mean (SD) & $5.4(2.7)$ & $5.2(2.6)$ & \multirow{3}{*}{$0.206^{1}$} \\
\hline Median $\left(25^{\text {th }}-75^{\text {th }}\right)$ & $5.1(3.5-7.8)$ & $4.5(3.2-6.9)$ & \\
\hline Minimum-maximum & $0.8-11.4$ & $1.0-11$ & \\
\hline
\end{tabular}

${ }^{1}$ Wilcoxon's test; ${ }^{2} \mathrm{McNemar}$ test; SD — standard deviation

Table 2. Analysis of agreement in the indication for uterine-artery embolization between clinical/ultrasound and pelvic MRI findings in women with fibroids

\begin{tabular}{|l|c|c|}
\hline \multirow{2}{*}{$\begin{array}{l}\text { Indication } \\
\text { Clinical/ultrasound }\end{array}$} & \multicolumn{2}{|c|}{ MRI indication } \\
\cline { 2 - 3 } No & No $(\mathbf{n}=\mathbf{6 2})$ & Yes $(\mathbf{n}=\mathbf{2 0 1})$ \\
\hline Yes & $29(46.8 \%)$ & $18(9.0 \%)$ \\
\hline
\end{tabular}

Kappa $=0.413(p<0.001) ; M R I-$ magnetic resonance imaging

Table 3. Agreement between ultrasound and magnetic resonance imaging for diagnosing adenomyosis in women referred for uterine-artery embolization

\begin{tabular}{|l|c|c|}
\multirow{2}{*}{ Diagnostic, ultrasound } & \multicolumn{2}{|c|}{$\begin{array}{c}\text { Diagnostic, magnetic resonance } \\
\text { imaging }\end{array}$} \\
\cline { 2 - 3 } & No $(\mathbf{n}=\mathbf{2 2 0})$ & Yes $(\mathbf{n}=\mathbf{4 3})$ \\
\hline No & $213(96.8 \%)$ & $38(88.4 \%)$ \\
\hline Yes & $7(3.2 \%)$ & $5(11.6 \%)$ \\
\hline
\end{tabular}

Kappa $=0.119(p=0.015)$; Sensitivity $=11.63 \%$ (US vs. MRI); Sensitivity $=96.82 \%$ (US vs. MRI); Accuracy $=82.9 \%$ (percentage of concordant pairs between the two methods); $\mathrm{MRI}=$ considered the gold standard

there was a statistically significant agreement between the two methods ( $p<0.001$ ), with a calculated kappa of 0.41 .

Of the 263 analyzed medical records, in 12 (4.56\%) women adenomyosis was observed in US. After evaluating MRI imaging, this diagnosis was ruled out in 7/12 cases. In addition to the five cases previously diagnosed by US, MRI examination showed adenomyosis in 38 other women, totaling 43/263 (16.35\%) cases. Table 3 shows the analysis of the agreement between US and MRI findings in the diagnosis of adenomyosis. As can be seen in the table, in 218/263 (82.9\%) of patients there was agreement between MRI and US findings for diagnosing adenomyosis, and adenomyosis classi- fication differed in 45 (17.1\%) of the women. We observed that the US diagnosed adenomyosis in seven women who were not diagnosed on MRI imaging. In view of these data, there was a statistically significant correlation between the two methods $(p<0.05)$, but the calculated kappa was very low $(<0.2)$.

\section{DISCUSSION}

In this study, the authors evaluated the experience of this service in analyzing 263 women who underwent uterine-artery embolization because of fibroids. The mean age of the women in the present study was lower than that observed by Laios et al. [12] (43.3 years) and by Rajan et al. [9] (43.4 years), who evaluated 118 and 180 women, respectively. In the present study, uterine volume was significantly higher in MRI than in US. This result was also observed by Stoelinga et al. [13], who assessed 83 women who underwent uterine-artery embolization because of fibroids, and observed a mean uterine volume $557 \mathrm{~cm}^{3}$ in MRI and $472 \mathrm{~cm}^{3}$ in US. Likewise, Rajan et al. [9] observed a mean volume of $701 \mathrm{~cm}^{3}$ in MRI and $658 \mathrm{~cm}^{3}$ in US. As for the number of fibroids, MRI showed a greater number of women with multiple fibroids. In a study conducted by Malartic et al. [2], US showed more than five fibroids in 12 women (12/68, $17.6 \%)$, whereas MRI showed more than five fibroids in 31 women $(31 / 68 ; 45.6 \%)$. It is not consensus in the literature regarding the importance of uterine volume and number of myomas as success factors for uterine artery embolization. Toor et al. [14] assessed 78 patients who underwent uterine-artery embolization to investigate the MRI findings associated with clinical failure. The reduction in uterine and dominant fibroid volumes was greater in the success group compared with the failure group; however, the difference was not statistically significant. The reduction in total fibroid 
volume was greater in the success group than the failure group $48.2 \%$ vs. $33.8 \%$, respectively.

In the present study, MRI altered the indication for uterine-artery embolization in 51/263 (19.4\%) cases, a result similar to that reported by Rajan et al. [9], who observed that the indication was altered in 14/116 (12.1\%) women because US did not correctly quantify/locate the fibroids. In the present study, adenomyosis was associated with fibroids in 43/263 (16.35\%) cases. The diagnosis of adenomyosis associated with fibroids is important for counseling women that the results of uterine-artery embolization, as well as the resolution of symptoms, may not be as long-lasting compared with the results obtained when the diagnosis is fibroid alone [15]. Of the 43 patients in the present study who were diagnosed with adenomyosis by MRI, only one did not have symptoms. In symptomatic cases, bleeding was present in $84 \%$ women aged $>35$ years. Bleeding is the main symptom, present in approximately $40-60 \%$ women [16].

Although US is the method of choice for diagnosing gynecological pelvic pathologies because of its low cost and greater accessibility, its sensitivity for diagnosing adenomyosis is low. In the present study, US had a sensitivity of $11.63 \%$ and specificity of $96.82 \%$, compared with MRI. In the study by Malartic et al. [2], US showed lower sensitivity (5.5\%) and similar specificity (98.3\%). However, in a systematic review Champaneria et al. [17] observed that US and MRI showed high and similar sensitivities ( $72 \%$ vs. $77 \%$, respectively) for diagnosing adenomyosis. Nonetheless, in the case of adenomyosis diagnosis using US is influenced by the examiner's experience, type of equipment, and anatomical presentation of the uterus; technical advances such as three-dimensional US can be an important tool in infertile women with adenomyosis [17]. In the present study, the authors believe that the low sensitivity of US resulted least partially from the fact that this examination was conducted in various imaging clinics with different examiners and equipments, however all examiners were board of Brazilian College of Radiology or Brazilian Federation of Gynecology and Obstetrics and the US equipments were high definition.

\section{CONCLUSIONS}

In summary, MRI was shown to be a better preoperative evaluation method for uterine-artery embolization because it modified the initial US diagnosis in $17.1 \%$ cases. US showed low sensitivity for diagnosing adenomyosis associated with fibroids in comparison with MRI.

\section{REFERENCES}

1. Baird DD, Dunson DB. Why is parity protective for uterine fibroids? Epidemiology. 2003; 14(2): 247-250, doi: 10.1097/01.EDE.0000054360.61254.27, indexed in Pubmed: 12606893.

2. Malartic C, Morel O, Rivain AL, et al. Evaluation of symptomatic uterine fibroids in candidates for uterine artery embolization: comparison between ultrasonographic and MR imaging findings in 68 consecutive patients. Clin Imaging. 2013; 37(1): 83-90, doi: 10.1016/j.clinimag.2012.03.007, indexed in Pubmed: 23206612.

3. Khan AT, Shehmar M, Gupta JK. Uterine fibroids: current perspectives. Int J Womens Health. 2014; 6: 95-114, doi: 10.2147/JJWH.S51083, indexed in Pubmed: 24511243.

4. Taran FA, Brown HL, Stewart EA. Racial diversity in uterine leiomyoma clinical studies. Fertil Steril. 2010; 94(4): 1500-1503, doi: 10.1016/j. fertnstert.2009.08.037, indexed in Pubmed: 19969296.

5. Omary RA, Vasireddy S, Chrisman HB, et al. The effect of pelvic MR imaging on the diagnosis and treatment of women with presumed symptomatic uterine fibroids. J Vasc Interv Radiol. 2002; 13(11): 1149-1153, indexed in Pubmed: 12427815.

6. Pelage JP, Le Dref O, Soyer P, et al. Fibroid-related menorrhagia: treatment with superselective embolization of the uterine arteries and midterm follow-up. Radiology. 2000; 215(2): 428-431, doi: 10.1148/radiology.215.2.r00ma11428, indexed in Pubmed: 10796920.

7. Chittawar PB, Kamath MS. Review of nonsurgical/minimally invasive treatments and open myomectomy for uterine fibroids. Curr Opin Obstet Gynecol. 2015; 27(6): 391-397, indexed in Pubmed: 26536205.

8. Early HM, McGahan JP, Scoutt LM, et al. Pitfalls of Sonographic Imaging of Uterine Leiomyoma. Ultrasound Q. 2016; 32(2): 164-174, indexed in Pubmed: 27233072

9. Rajan DK, Margau R, Kroll RR, et al. Clinical utility of ultrasound versus magnetic resonance imaging for deciding to proceed with uterine artery embolization for presumed symptomatic fibroids. Clin Radiol. 2011;66(1): 57-62, doi: 10.1016/j.crad.2010.08.005, indexed in Pubmed: 21147300.

10. Devlieger R, $\mathrm{D}^{\prime}$ Hooghe $\mathrm{T}$, Timmerman $\mathrm{D}$. Uterine adenomyosis in the infertility clinic. Hum Reprod Update. 2003; 9(2): 139-147, indexed in Pubmed: 12751776.

11. Stamatopoulos CP, Mikos T, Grimbizis GF, et al. Value of magnetic resonance imaging in diagnosis of adenomyosis and myomas of the uterus. J Minim Invasive Gynecol. 2012; 19(5): 620-626, doi: 10.1016/j. jmig.2012.06.003, indexed in Pubmed: 22935303.

12. Laios $A$, Baharuddin N, lliou K, et al. Uterine artery embolization for treatment of symptomatic fibroids; a single institution experience. Hippokratia. 2014; 18(3): 258-261, indexed in Pubmed: 25694762.

13. Stoelinga $B$, Huirne J, Heymans MW, et al. The estimated volume of the fibroid uterus: a comparison of ultrasound and bimanual examination versus volume at MRI or hysterectomy. Eur J Obstet Gynecol Reprod Biol. 2015; 184: 89-96, doi: 10.1016/j.ejogrb.2014.11.011, indexed in Pubmed: 25481364.

14. Toor SS, Tan KT, Simons ME, et al. Clinical failure after uterine artery embolization: evaluation of patient and MR imaging characteristics. J VasC Interv Radiol. 2008; 19(5): 662-667, doi: 10.1016/j.jvir.2007.12.454, indexed in Pubmed: 18440453.

15. Silberzweig JE, Powell DK, Matsumoto $\mathrm{AH}$, et al. Management of Uterine Fibroids: A Focus on Uterine-sparing Interventional Techniques. Radiology. 2016; 280(3): 675-692, doi: 10.1148/radiol.2016141693, indexed in Pubmed: 27533290.

16. Struble J, Reid S, Bedaiwy MA. Adenomyosis: A Clinical Review of a Challenging Gynecologic Condition. J Minim Invasive Gynecol. 2016; 23(2): 164-185, doi: 10.1016/j.jmig.2015.09.018, indexed in Pubmed: 26427702.

17. Champaneria R, Abedin P, Daniels J, et al. Ultrasound scan and magnetic resonance imaging for the diagnosis of adenomyosis: systematic review comparing test accuracy. Acta Obstet Gynecol Scand. 2010; 89(11): 1374-1384, doi: 10.3109/00016349.2010.512061, indexed in Pubmed: 20932128.

18. Puente JM, Fabris A, Patel J, et al. Adenomyosis in infertile women prevalence and the role of $3 \mathrm{D}$ ultrasound as a marker of severity of the disease. Reprod Biol Endocrinol. 2016; 14(1): 60, doi: 10.1186/s12958016-0185-6, indexed in Pubmed: 27645154. 